\title{
Kantorovich-Vlasov Method for the Flexural Analysis of Kirchhoff Plates with Opposite Edges Clamped, and Simply Supported (CSCS Plates)
}

\author{
Onah H.N. $^{\# 1}$, Mama B.O. ${ }^{\# 2}$, Ike, C.C. ${ }^{* 3}$, Nwoji, C.U. ${ }^{4}$ \\ \#1, \#2, \#4 Dept of Civil Engineering \\ ${ }^{* 3}$ University of Nigeria, Nsukka, Enugu State, Nigeria. \\ Enugu State University of Science \& Technology, Enugu State, Nigeria. \\ ${ }^{1}$ hyginus.onah@unn.edu.ng \\ 2benjamin.mama@unn.edu.ng \\ 3ikecc2007@yahoo.com \\ 4clifford.nwoji@unn.edu.ng
}

\begin{abstract}
The Kantorovich-Vlasov method was used, in this study, for the flexural analysis of rectangular Kirchhoff plates with opposite edges $(x=0$, and $x=a)$ simply supported and the other opposite edges $(y=$ 0 , and $y=b$ ) clamped (CSCS plates). The plate was subjected to a linear distribution of load over the entire plate domain. Vlasov method was used in finding the coordinate function in the $x$-direction, and Kantorovich method was used to consider the displacement function for the plate. The total potential energy functional, and the corresponding Euler-Lagrange differential equations were then obtained for the plate problem. This was solved subject to the boundary conditions in the $y$ direction to obtain the displacement function which minimized the total potential energy functional. Bending moment distributions were obtained using the bending moment-displacement equations. The solutions obtained for deflection and bending moment distributions were found to be rapidly convergent single series. Deflections and bending moment computed at the center of the plate were also rapidly convergent series. The solutions obtained for deflections and bending moments $\left(M_{x x}\right.$ and $\left.M_{y y}\right)$ were exactly identical with solutions presented by Timoshenko and Woinowsky-Krieger who used the method of superposition.
\end{abstract}

Keywords: Kantorovich-Vlasov method, Kirchhoff plate, total potential energy functional, Euler-Lagrange differential equation.

\section{INTRODUCTION}

Plates are structural members characterized by a transverse dimension that is much smaller than the other inplane dimensions. They are used in civil, mechanical, aeronautical and marine engineering to model roof and floor slabs, ship hulls, bridge deck slabs, spacecraft panels, retaining walls, foundations slabs, and aircraft panels $[1,2,3]$. Plates may be subject to transverse static or dynamic loads or inplane compressive loads resulting in static flexure, dynamic flexure or buckling behaviours. They are classified according to shapes as: rectangular, circular, elliptical, rhombic, skew, or according to the ratio of their thickness to the least inplane dimension as thin plates, moderately thick plates and thick plates $[1,2,3]$. They are also classified based on the material elastic properties as homogeneous, heterogeneous, isotropic, anisotropic, and orthotropic.

Several theories have been used to describe the response and behaviours of plates under loads. They are: Kirchhoff plate theory which is suitable for thin plates, Reissner [4] plate theory, Mindlin [5] plate theory, Levinson's shear deformation plate theories, Reddy's plate theory, Shimpi's refined plate theory [6], [7]. Theory of elasticity methods have been also used to describe plate behaviour. Kirchhoff plate theory, also called the classical plate theory has been found to be suitable for describing the behaviours of thin plates under static flexure, dynamic flexure and buckling; and is adopted in this study. Reissner's stress based theory and Mindlin's first order shear deformation theory are suitable for moderately thick plates and take account of the effect of shear deformation on the plate response under transverse loads. Other theories that take account of the shear deformation of the plate and thus are suitable for describing moderately thick plates include shear deformation plate theories proposed by Levinson; Reddy, and Shimpi's refined plate theories. Thick plates are described using the theories of elasticity for three dimensional bodies. 


\section{METHODS OF SOLVING PLATE PROBLEMS}

Plate problems as described by various theories are boundary value problems. Two broad methods of solving boundary value problems of plates are: mathematical or analytical methods, and numerical or approximate methods. Mathematical or analytical methods are methods used to obtain solutions that satisfy the governing differential equations of the plate problem at all points on the plate domain, and on the plate boundaries. They result in closed form solutions. They include: Navier [8] method, Levy [9] method, separation of variables method, integral transform methods. Numerical methods are methods that aim to obtain approximate solutions to the governing boundary value problem of plates. They include: finite difference methods [10], finite element methods, boundary element methods, variational Ritz methods [11], variational Galerkin method [12, 13], collocation methods, Bubnov-Galerkin method, Kantorovich method [14, 15], Vlasov method [14, 15].

\section{RESEARCH AIM AND OBJECTIVES}

The aim of this study is to apply the Kantorovich-Vlasov method to the analysis of Kirchhoff CSCS plates under linearly distributed transverse loads on the entire plate domain. The specific objectives are:

(i) to obtain the total potential energy functional $\Pi$ for the CSCS Kirchhoff plate bending problem based on shape functions obtained by Kantorovich and Vlasov approaches.

(ii) to obtain the Euler-Lagrange-Ostragradsky differential equations for the extremization of the total potential energy functional.

(iii) to solve the Euler-Lagrange-Ostragradsky differential equations subject to the boundary conditions of the plate.

(iv) to obtain the bending moment distributions.

(v) to obtain the deflections and bending moments at the center of the plate for various plate aspect ratios.

\section{Theoretical Framework and Methodology}

The Kantorovich-Vlasov method is a variational method based on finding the displacement function that minimizes the total potential energy functional. The displacement function is assumed to be in variableseparable form as the sum of the product of coordinate functions in the $x$ and $y$ directions, with the coordinate function in one of the directions chosen as the eigenfunctions of a vibrating Euler-Bernoulli beam with equivalent end supports as the plate. The total potential energy functional $\Pi$ for a rectangular Kirchhoff plate under applied load is given by:

$$
\Pi=\frac{D}{2} \iint_{R}\left\{\left(\frac{\partial^{2} w}{\partial x^{2}}+\frac{\partial^{2} w}{\partial y^{2}}\right)^{2}+2(1-\mu)\left(\frac{\partial^{2} w}{\partial x \partial y}\right)^{2}-2(1-\mu) \frac{\partial^{2} w}{\partial x^{2}} \frac{\partial^{2} w}{\partial y^{2}}\right\} d x d y-\iint_{R} p w(x, y) d x d y
$$

where $R$ is the plate domain, $w(x, y)$ is the transverse deflection of the plate, $p(x, y)$ is the transverse load distribution, $\mu$ is the Poisson's ratio, $x$ and $y$ are the inplane Cartesian coordinates and $D$ is the flexural rigidity of the plate.

In the Kantorovich-Vlasov method, the deflection function $w(x, y)$ is assumed as the infinite series:

$$
w(x, y)=\sum_{n=1}^{\infty} g_{n}(y) f_{n}(x)
$$

where either $g_{n}(y)$ or $f_{n}(x)$ is known to be the eigenfunctions of vibrating Euler-Bernoulli beam with the same end supports as the plate in the corresponding coordinate direction; and the unknown function sought in order to minimize the total potential energy functional $\Pi$.

\section{Application of Kantorovich-Vlasov method Problem description}

Consider a rectangular Kirchhoff plate with inplane dimensions $a \times b$, simply supported on the opposite edges $x=0$, and $x=a$, and clamped on the other opposite edges $y=0$ and $y=b$, as shown in Figure 1 . 


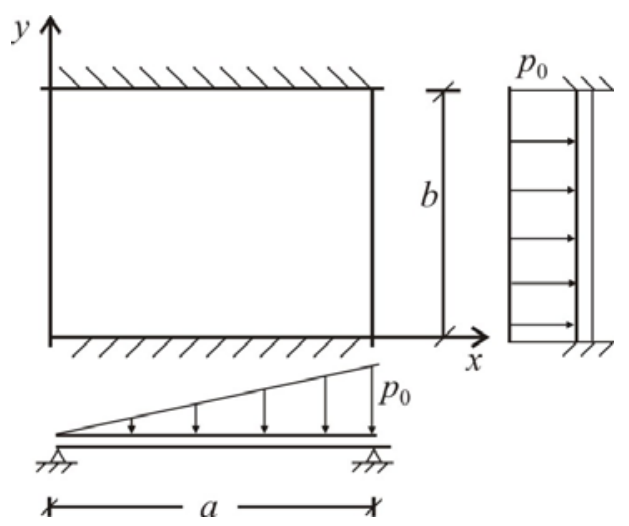

Figure 1: Rectangular Kirchhoff plate, with opposite edges clamped and simply supported under linear distributed transverse load

The entire plate domain is subjected to linearly distributed transverse load of intensity $p(x)=p_{0} x / a$

\section{DEFLECTION FUNCTION}

Following Kantorovich procedure, the deflection function $w(x, y)$ is assumed to be a linear combination of the sum of products of linearly independent coordinate (shape or basis) functions as Equation (2). The coordinate functions $f_{n}(x)$ in the $x$-direction are chosen by the Vlasov procedure as the vibrating modal shape functions of a freely vibrating prismatic Euler-Bernoulli beam simply supported at $x=0$, and $x=a$. For simply supported Euler-Bernoulli beams, the eigenfunctions are:

$$
f_{n}(x)=\sin \frac{n \pi x}{a}
$$

$n=1,2,3,4, \ldots \infty$

Then the deflection function becomes:

$$
w(x, y)=\sum_{n=1}^{\infty} g_{n}(y) \sin \frac{n \pi x}{a}
$$

\section{TOTAL POTENTIAL ENERGY FUNCTIONAL}

By substitution of Equation (4) into the total potential energy functional, we obtain:

$$
\begin{aligned}
\Pi=\frac{D}{2} \iint_{R}\left\{\left[\sum_{n=1}^{\infty}-\left(\frac{n \pi}{a}\right)^{2} g_{n}(y) \sin \frac{n \pi x}{a}+\sum_{n=1}^{\infty}\left(g_{n}^{\prime \prime}(y) \sin \frac{n \pi x}{a}\right)\right]^{2}\right. \\
+2(1-\mu)\left(\sum_{n=1}^{\infty} \frac{n \pi}{a} g_{n}^{\prime}(y) \cos \frac{n \pi x}{a}\right)^{2}-2(1-\mu)\left(\sum_{n=1}^{\infty}-\left(\frac{n \pi}{a}\right)^{2} g_{n}(y) \sin \frac{n \pi x}{a}\right) \\
\left.\times\left(\sum_{n=1}^{\infty} g_{n}^{\prime \prime}(y) \sin \frac{n \pi x}{a}\right)\right\} d x d y-\iint_{R}\left(p(x, y) \sum_{n=1}^{\infty} g_{n}(y) \sin \frac{n \pi x}{a}\right) d x d y
\end{aligned}
$$

The load distribution is expressed in terms of single sine series to obtain:

$$
p(x, y)=\sum_{n=1}^{\infty} p_{n} \sin \frac{n \pi x}{a}
$$

Using Equation (6) and simplifying the total potential energy expression in Equation (5), we obtain:

$$
\begin{aligned}
\Pi=\sum_{n=1}^{\infty} & \frac{D}{2}\left\{\int_{0}^{a} \int_{0}^{b}\left(\left(\frac{n \pi}{a}\right)^{4}\left(g_{n}(y)\right)^{2} \sin ^{2} \frac{n \pi x}{a}+\left(g_{n}^{\prime \prime}(y)\right)^{2} \sin ^{2} \frac{n \pi x}{a}-\frac{2 n \pi}{a} g_{n}(y) g_{n}^{\prime \prime}(y) \sin ^{2} \frac{n \pi x}{a}\right)\right. \\
& \left.+2(1-\mu)\left(\frac{n \pi}{a}\right)^{2}\left(g_{n}^{\prime}(y)\right)^{2} \cos ^{2} \frac{n \pi x}{a}-2(1-\mu)\left(-\left(\frac{n \pi}{a}\right)^{2} g_{n}(y) g_{n}^{\prime \prime}(y) \sin ^{2} \frac{n \pi x}{a}\right)\right\} d x d y \\
& -\sum_{n=1}^{\infty} \int_{0}^{a} \int_{0}^{b} p_{n}(y) g_{n}(y) \sin ^{2} \frac{n \pi x}{a} d x d y
\end{aligned}
$$


Simplifying further,

$$
\begin{aligned}
\Pi=\sum_{n=1}^{\infty} & \frac{D}{2} \frac{a}{2} \int_{0}^{b}\left(\left(\frac{n \pi}{a}\right)^{4}\left(g_{n}(y)\right)^{2}+\left(g_{n}^{\prime \prime}(y)\right)^{2}-2\left(\frac{n \pi}{a}\right)^{2} g_{n}(y) g_{n}^{\prime \prime}(y)+2(1-\mu)\left(\frac{n \pi}{a}\right)^{2}\left(g_{n}^{\prime}(y)\right)^{2}\right. \\
& \left.+2(1-\mu)\left(\frac{n \pi}{a}\right)^{2} g_{n}(y) g_{n}^{\prime \prime}(y)\right) d y-\frac{a}{2} \int_{0}^{b} p_{n}(y) g_{n}(y) d y
\end{aligned}
$$

Alternatively,

$$
\begin{aligned}
\Pi=\sum_{n=1}^{\infty} & \frac{D a}{4}\left\{\int _ { 0 } ^ { b } \left(\left(\frac{n \pi}{a}\right)^{4}(g(y))^{2}+\left(g^{\prime \prime}(y)\right)^{2}-2\left(\frac{n \pi}{a}\right)^{2} g_{n}(y) g_{n}^{\prime \prime}(y)+2(1-\mu)\left(\frac{n \pi}{a}\right)^{2}\left(g_{n}^{\prime}(y)\right)^{2}\right.\right. \\
& \left.\left.+2(1-\mu)\left(\frac{n \pi}{a}\right)^{2} g_{n}(y) g_{n}^{\prime \prime}(y)-\frac{2}{D} p_{n}(y) g_{n}(y)\right) d y\right\}
\end{aligned}
$$

Equation (9) is the total potential energy functional for the plate whose minimum with respect to $g(y)$ we wish to find, in order to fully solve the Kirchhoff plate flexure problem. The integrand $F\left(y, g(y) g^{\prime}(y) g^{\prime \prime}(y)\right)$ is

$$
\begin{aligned}
& F\left(y, g(y) g^{\prime}(y), g^{\prime \prime}(y)\right)=\left(\frac{n \pi}{a}\right)^{4}\left(g_{n}(y)\right)^{2}+\left(g^{\prime \prime}(y)\right)^{2}-2\left(\frac{n \pi}{a}\right)^{2} g_{n}(y) g_{n}^{\prime \prime}(y) \\
& +2(1-\mu)\left(\frac{n \pi}{a}\right)^{2} g^{\prime}(y)+2(1-\mu)\left(\frac{n \pi}{a}\right)^{2} g_{n}(y) g_{n}^{\prime \prime}(y)-\frac{2}{D} p_{n}(y) g_{n}(y)
\end{aligned}
$$

\section{EULER-LAGRANGE-OSTRAGRADSKY DIFFERENTIAL EQUATION}

The Euler-Lagrange-Ostragradsky differential equation of equilibrium which represents the condition for a minimum of the total potential energy functional $\Pi$ is given by:

$$
-\frac{\partial F}{\partial g_{n}(y)}+\frac{d}{d y}\left(\frac{\partial F}{\partial g_{n}^{\prime}(y)}\right)-\frac{d^{2}}{d y^{2}}\left(\frac{\partial F}{\partial g^{\prime \prime}(y)}\right)=0
$$

Applying Equation (11) we obtain:

$$
\begin{aligned}
-2 g_{n}^{i v} & +2 \mu\left(\frac{n \pi}{a}\right)^{2} g_{n}^{\prime \prime}(y)+4(1-\mu)\left(\frac{n \pi}{a}\right)^{2} g_{n}^{\prime \prime}(y)-2\left(\frac{n \pi}{a}\right)^{4} g_{n}(y) \\
& +2\left(\frac{n \pi}{a}\right)^{2} g_{n}^{\prime \prime}(y)-2(1-\mu)\left(\frac{n \pi}{a}\right)^{2} g_{n}^{\prime \prime}(y)+\frac{2}{D} p_{n}(y)=0
\end{aligned}
$$

Simplification yields:

$$
g_{n}^{i v}(y)-2\left(\frac{n \pi}{a}\right)^{2} g_{n}^{\prime \prime}(y)+\left(\frac{n \pi}{a}\right)^{4} g_{n}(y)=\frac{p_{n}(y)}{D}
$$

Equation (13) is a fourth order linear ordinary differential equation in $g_{n}(y)$ as unknown; and is solved subject to the boundary conditions of the plate support at the edges $y=0$ and $y=b$.

\section{LOAD DISTRIBUTION SERIES COEFFICIENT $P_{N}$}

The single Fourier sine series coefficient for the hydrostatic load distribution is, from single Fourier sine series theory,

$$
\begin{aligned}
p_{n} & =\frac{2}{a} \int_{0}^{a} p(x, y) \sin \frac{n \pi x}{a} d x \\
& =\frac{2}{a} \int_{0}^{a} \frac{p_{0} x}{a} \sin \frac{n \pi x}{a} d x \\
& =\frac{2 p_{0}}{a^{2}} \int_{0}^{a} x \sin \frac{n \pi x}{a} d x
\end{aligned}
$$




$$
p_{n}=\frac{2 p_{0}}{a^{2}} \cdot \frac{-a^{2} \cos n \pi}{n \pi}=\frac{(-1)^{n+1} 2 p_{0}}{n \pi}
$$

\section{SOLUTION OF THE EULER-LAGRANGE-OSTRAGRADSKY EQUATION}

Using equation (17), the Euler-Lagrange-Ostragradsky differential equation for the Kirchhoff plate problem becomes:

$$
g_{n}^{i v}(y)-2\left(\frac{n \pi}{a}\right)^{2} g_{n}^{\prime \prime}(y)+\left(\frac{n \pi}{a}\right)^{4} g_{n}(y)=\frac{(-1)^{n+1} 2 p_{0}}{n \pi D}
$$

The homogeneous solution is the solution to the fourth order homogeneous linear ordinary differential equation (ODE):

$$
g_{n}^{i v}(y)-2\left(\frac{n \pi}{a}\right)^{2} g_{n}^{\prime \prime}(y)+\left(\frac{n \pi}{a}\right)^{4} g_{n}(y)=0
$$

Using the method of trial functions, it is assumed that the homogeneous solution to $g_{n}(y)$ is in the form of the exponential function:

$$
g_{n}(y)=\exp s y=e^{s y}
$$

where $s$ is an undetermined parameter we seek to determine.

Then, the homogeneous ODE becomes:

$$
s^{4} e^{s y}-2\left(\frac{n \pi}{a}\right)^{2} s^{2} e^{s y}+\left(\frac{n \pi}{a}\right)^{4} e^{s y}=0
$$

Simplifying,

$$
\left(s^{4}-2\left(\frac{n \pi}{a}\right)^{2} s^{2}+\left(\frac{n \pi}{a}\right)^{4}\right) e^{s y}=0
$$

For non-trivial solutions,

$$
e^{s y} \neq 0
$$

The auxiliary (characteristic) polynomial is:

$$
\begin{aligned}
& s^{4}-2\left(\frac{n \pi}{a}\right)^{2} s^{2}+\left(\frac{n \pi}{a}\right)^{4}=0 \\
& \left(s^{2}-\left(\frac{n \pi}{a}\right)^{2}\right)^{2}=0
\end{aligned}
$$

The roots are

$$
\begin{array}{ll}
s_{1,2}=+\frac{n \pi}{a} & \text { double root } \\
s_{3,4}=-\frac{n \pi}{a} & \text { double root }
\end{array}
$$

The homogeneous solution then becomes:

$$
g_{n}(y)=c_{1_{n}} \exp \frac{n \pi y}{a}+c_{2_{n}} \exp -\frac{n \pi y}{a}+c_{3_{n}} y \exp \frac{n \pi y}{a}+c_{4_{n}} y \exp -\frac{n \pi y}{a}
$$

where $c_{1_{n}}, c_{2_{n}}, c_{3_{n}}$ and $c_{4_{n}}$ are the integration constants.

From the distributed load which is not a function of $y$, we consider that particular solutions $g_{n p}$ would not depend upon $y$ and thus:

$$
g_{n p}^{\prime \prime}=g_{n p}^{i v}=0
$$

Then, by substitution into the inhomogeneous ODE, Equation (18), we obtain, after simplification;

$$
g_{n p}=\frac{(-1)^{n+1} 2 p_{0} a^{4}}{(n \pi)^{5} D}
$$


The general solution is obtained by using the superposition (linearity) principle as the sum of the homogeneous and particular solutions, as follows:

$$
g_{n}(y)=c_{1_{n}} \exp \frac{n \pi y}{a}+c_{2_{n}} \exp -\frac{n \pi y}{a}+c_{3_{n}} y \exp \frac{n \pi y}{a}+c_{4_{n}} y \exp -\frac{n \pi y}{a}+\frac{(-1)^{n+1} 2 p_{0} a^{4}}{(n \pi)^{5} D}
$$

\section{ENFORCEMENT OF BOUNDARY CONDITIONS}

The boundary conditions at the clamped edges $y=0$, and $y=b$ are the four conditions for each value of $n$ :

$$
\begin{aligned}
& g_{n}(y=0)=0 \\
& g_{n}(y=b)=0 \\
& g_{n}^{\prime}(y=0)=0 \\
& g_{n}^{\prime}(y=b)=0
\end{aligned}
$$

The enforcement/application of the four boundary conditions on $g_{n}(y)$ results in a system of four equations in terms of the four integration constants for each $n$ value. These four equations are then solved simultaneously to obtain the four constants of integration $c_{1_{n}}, c_{2_{n}}, c_{3_{n}}$ and $c_{4_{n}}$ at the $n$th term. Enforcement of the boundary conditions yield:

$$
\begin{aligned}
& c_{1_{n}}+c_{2_{n}}=\frac{(-1)^{n} 2 p_{0} a^{4}}{(n \pi)^{5} D} \\
& \alpha_{n} c_{1_{n}}-\alpha_{n} c_{2_{n}}+c_{3_{n}}+c_{4_{n}}=0 \\
& c_{1_{n}} e^{\alpha_{n} b}+c_{2_{n}} e^{-\alpha_{n} b}+c_{3_{n}} b e^{\alpha_{n} b}+c_{4_{n}} b e^{-\alpha_{n} b}=\frac{(-1)^{n} 2 p_{0} a^{4}}{(n \pi)^{5} D} \\
& c_{1_{n}} \alpha_{n} e^{\alpha_{n} b}-\alpha_{n} c_{2_{n}} e^{-\alpha_{n} b}+c_{3_{n}}\left(1+\alpha_{n} b\right) e^{\alpha_{n} b}+c_{4_{n}}\left(1-\alpha_{n} b\right) e^{-\alpha_{n} b}=0
\end{aligned}
$$

where

$$
\alpha_{n}=n \pi / a
$$

Let

$$
\bar{p}_{n}=\frac{(-1)^{n} 2 p_{0} a^{4}}{(n \pi)^{5} D}
$$

Then, the system of four algebraic equations can be expressed in matrix form as:

$$
\left(\begin{array}{cccc}
1 & 1 & 0 & 0 \\
\alpha_{n} & -\alpha_{n} & 1 & 1 \\
e^{\alpha_{n} b} & e^{-\alpha_{n} b} & b e^{\alpha_{n} b} & b e^{-\alpha_{n} b} \\
\alpha_{n} e^{\alpha_{n} b} & -\alpha_{n} e^{-\alpha_{n} b} & \left(1+\alpha_{n} b\right) e^{\alpha_{n} b} & \left(1-\alpha_{n} b\right) e^{-\alpha_{n} b}
\end{array}\right)\left(\begin{array}{c}
c_{1_{n}} \\
c_{2_{n}} \\
c_{3_{n}} \\
c_{4_{n}}
\end{array}\right)=\bar{p}_{n}\left(\begin{array}{l}
1 \\
0 \\
1 \\
0
\end{array}\right)
$$

This is solved using Cramer's rule to obtain the four constants as follows:

$$
\begin{aligned}
& c_{1_{n}}=\frac{D_{1}}{D_{0}} \\
& c_{2_{n}}=\frac{D_{2}}{D_{0}} \\
& c_{3_{n}}=\frac{D_{3}}{D_{0}} \\
& c_{4_{n}}=\frac{D_{4}}{D_{0}}
\end{aligned}
$$

where 


$$
\begin{aligned}
& D_{0}=\left|\begin{array}{cccc}
1 & 1 & 0 & 0 \\
\alpha_{n} & -\alpha_{n} & 1 & 1 \\
e^{\alpha_{n} b} & e^{-\alpha_{n} b} & b e^{\alpha_{n} b} & b e^{-\alpha_{n} b} \\
\alpha_{n} e^{\alpha_{n} b} & -\alpha_{n} e^{-\alpha_{n} b} & \left(1+\alpha_{n} b\right) e^{\alpha_{n} b} & \left(1-\alpha_{n} b\right) e^{-\alpha_{n} b}
\end{array}\right| \\
& D_{1}=\left|\begin{array}{cccc}
\bar{p}_{n} & 1 & 0 & 0 \\
0 & \alpha_{n} & 1 & 1 \\
\bar{p}_{n} & e^{-\alpha_{n} b} & b e^{\alpha_{n} b} & b e^{-\alpha_{n} b} \\
0 & -\alpha_{n} e^{-\alpha_{n} b} & \left(1+\alpha_{n} b\right) e^{\alpha_{n} b} & \left(1-\alpha_{n} b\right) e^{-\alpha_{n} b}
\end{array}\right| \\
& D_{2}=\left|\begin{array}{cccc}
1 & \bar{p}_{n} & 0 & 0 \\
\alpha_{n} & 0 & 1 & 1 \\
e^{\alpha_{n} b} & \bar{p}_{n} & b e^{\alpha_{n} b} & b e^{-\alpha_{n} b} \\
\alpha_{n} e^{\alpha_{n} b} & 0 & \left(1+\alpha_{n} b\right) e^{\alpha_{n} b} & \left(1-\alpha_{n} b\right) e^{-\alpha_{n} b}
\end{array}\right| \\
& D_{3}=\left|\begin{array}{cccc}
1 & 1 & \bar{p}_{n} & 0 \\
\alpha_{n} & \alpha_{n} & 0 & 1 \\
e^{\alpha_{n} b} & e^{-\alpha_{n} b} & \bar{p}_{n} & b e^{-\alpha_{n} b} \\
\alpha_{n} e^{\alpha_{n} b} & -\alpha_{n} e^{-\alpha_{n} b} & 0 & \left(1-\alpha_{n} b\right) e^{-\alpha_{n} b}
\end{array}\right| \\
& D_{4}=\left|\begin{array}{cccc}
1 & 1 & 0 & \bar{p}_{n} \\
\alpha_{n} & \alpha_{n} & 1 & 0 \\
e^{\alpha_{n} b} & e^{-\alpha_{n} b} & b e^{\alpha_{n} b} & \bar{p}_{n} \\
\alpha_{n} e^{\alpha_{n} b} & -\alpha_{n} e^{-\alpha_{n} b} & \left(1+\alpha_{n} b\right) e^{\alpha_{n} b} & 0
\end{array}\right|
\end{aligned}
$$

Thus,

$$
\begin{aligned}
& c_{1_{n}}=\frac{\bar{p}_{n}\left(1+2 \alpha_{n} b+2\left(\alpha_{n} b\right)^{2}-e^{-\alpha_{n} b}\left(1+\alpha_{n} b\right)+e^{-\alpha_{n} b}\left(1-\alpha_{n} b\right)-e^{-2 \alpha_{n} b}\right)}{2+4\left(\alpha_{n} b\right)^{2}-e^{2 \alpha_{n} b}-e^{-2 \alpha_{n} b}} \\
& c_{2_{n}}=\frac{\bar{p}_{n}\left(-e^{-\alpha_{n} b}\left(1-\alpha_{n} b\right)+e^{\alpha_{n} b}\left(1+\alpha_{n} b\right)-e^{-2 \alpha_{n} b}-2 \alpha_{n} b+2\left(\alpha_{n} b\right)^{2}\right)}{2+4\left(\alpha_{n} b\right)^{2}-e^{2 \alpha_{n} b}-e^{-2 \alpha_{n} b}} \\
& c_{3_{n}}=\frac{\bar{p}_{n} \alpha_{n}\left(e^{-\alpha_{n} b}\left(2 \alpha_{n} b-1\right)+e^{-2 \alpha_{n} b}+e^{\alpha_{n} b}-1-2 \alpha_{n} b\right)}{2+4\left(\alpha_{n} b\right)^{2}-e^{2 \alpha_{n} b}-e^{-2 \alpha_{n} b}} \\
& c_{4_{n}}=\frac{\bar{p}_{n} \alpha_{n}\left(e^{\alpha_{n} b}\left(1+2 \alpha_{n} b\right)-e^{-\alpha_{n} b}-e^{2 \alpha_{n} b}+1-2 \alpha_{n} b\right)}{2+4\left(\alpha_{n} b\right)^{2}-e^{2 \alpha_{n} b}-e^{-2 \alpha_{n} b}}
\end{aligned}
$$

Then,

$$
w(x, y)=\sum_{n=1}^{\infty}\left(c_{1_{n}} e^{\alpha_{n} y}+c_{2_{n}} e^{-\alpha_{n} y}+c_{3_{n}} y e^{-\alpha_{n} y}+c_{4_{n}} y e^{-\alpha_{n} y}+\bar{p}_{n}\right) \sin \alpha_{n} x
$$

These constants of integration are expressed in terms of the aspect ratio $b / a=r$, as follows:

$$
\begin{aligned}
& c_{1_{n}}=\frac{\bar{p}_{n}\left(1+2 n \pi r+2(n \pi r)^{2}-e^{-n \pi r}(1+n \pi r)+e^{-n \pi r}(1-n \pi r)-e^{-2 n \pi r}\right)}{2+4(n \pi r)^{2}-e^{2 n \pi r}-e^{-2 n \pi r}} \\
& c_{2_{n}}=\frac{\bar{p}_{n}\left(-e^{-n \pi r}(1-n \pi r)+e^{n \pi r}(1+n \pi r)-e^{-2 n \pi r}-2 n \pi r+2(n \pi r)^{2}\right)}{2+4(n \pi r)^{2}-e^{2 n \pi r}-e^{-2 n \pi r}} \\
& c_{3_{n}}=\frac{\bar{p}_{n} \alpha_{n}\left(e^{-n \pi r}(2 n \pi r-1)+e^{-2 n \pi r}+e^{n \pi r}-1-2 n \pi r\right)}{2+4(n \pi r)^{2}-e^{2 n \pi r}-e^{-2 n \pi r}}
\end{aligned}
$$




$$
c_{4_{n}}=\frac{\bar{p}_{n} \alpha_{n}\left(e^{n \pi r}(1+2 n \pi r)-e^{-n \pi r}-e^{2 n \pi r}+1-2 n \pi r\right)}{2+4(n \pi r)^{2}-e^{2 n \pi r}-e^{-2 n \pi r}}
$$

\section{BENDING MOMENT DISTRIBUTIONS}

The bending moment distributions are obtained from the bending moment deflection equations:

$$
\begin{aligned}
& M_{x x}=-D\left(w_{x x}+\mu w_{y y}\right) \\
& M_{y y}=-D\left(w_{y y}+\mu w_{x x}\right)
\end{aligned}
$$

where

$$
w_{x x}=\frac{\partial^{2} w}{\partial x^{2}}=\sum_{n=1}^{\infty}-\alpha_{n}^{2} \sin \alpha_{n} x\left(c_{1_{n}} e^{\alpha_{n} y}+c_{2_{n}} e^{-\alpha_{n} y}+c_{3_{n}} y e^{\alpha_{n} y}+c_{4_{n}} y e^{-\alpha_{n} y}+\bar{p}_{n}\right)
$$

and

$$
w_{x x}=\frac{\partial^{2} w}{\partial y^{2}}=\sum_{n=1}^{\infty}\left(c_{1_{n}} \alpha_{n}^{2} e^{\alpha_{n} y}+c_{2_{n}} \alpha_{n}^{2} e^{-\alpha_{n} y}+c_{3_{n}}\left(\alpha_{n}^{2} y e^{\alpha_{n} y}+2 \alpha_{n} e^{\alpha_{n} y}\right)+c_{4_{n}}\left(\alpha_{n}^{2} y e^{-\alpha_{n} y}-2 \alpha_{n} e^{-\alpha_{n} y}\right)\right) \sin \alpha_{n} x
$$

The deflection equation, Equation (56) is used to determine the deflection of the center of square Kirchhoff plate under hydrostatic loads for increasing integer values of $n$, and the results are tabulated in Table 1 . The table reveals the rapidly convergent property of the series with satisfactory results obtained with $n=3$. Table 2 shows the converged results for the deflection and bending moment at the center of square Kirchhoff plate under hydrostatic load. The convergence study of the series for bending moments is shown in Table 3. Table 4 shows the variation of center bending moments $M_{x x}$, and $M_{y y}$ with the plate aspect ratio, while Table 5 presents the variation of center deflection with plate aspect ratio.

TABLE 1: Convergence study for center deflections of square Kirchhoff (CSCS) plate under hydrostatic load

\begin{tabular}{|c|c|}
\hline Number of terms, $n$ & Center deflection $w_{c} \times 10^{-4} p_{0} a^{4} / D$ \\
\hline 1 & 9.8093 \\
\hline 2 & 9.8093 \\
\hline 3 & 9.5679 \\
\hline 4 & 9.5679 \\
\hline 5 & 9.5887 \\
\hline 6 & 9.5887 \\
\hline 7 & 9.5848 \\
\hline 8 & 9.5848 \\
\hline 9 & 9.5859 \\
\hline 10 & 9.5859 \\
\hline 11 & 9.5855 \\
\hline 12 & 9.5855 \\
\hline
\end{tabular}

TABLE 2: Converged results for deflections and bending moments at the center of square Kirchhoff (CSCS) plates under hydrostatic load

\begin{tabular}{|c|c|c|c|}
\hline$a / b$ & $w_{c} \times p_{0} a^{4} / D$ & $M_{x x} \times p_{0} a^{2}$ & $M_{y y} \times p_{0} a^{2}$ \\
\hline 1 & $9.5855 \times 10^{-4}$ & $1.2176 \times 10^{-2}$ & $1.6617 \times 10^{-2}$ \\
\hline
\end{tabular}


TABLE 3: Convergence study for bending moment coefficients at the center of square Kirchhoff CSCS plate under hydrostatic load.

$$
p(x)=p_{0} x / a \quad(\mu=0.30)
$$

\begin{tabular}{|c|c|c|}
\hline Number of terms, $n$ & $M_{x x}=\beta_{x x} p_{0} a^{2}$ & $M_{y y}=\beta_{y y} p_{0} a^{2}$ \\
\hline 1 & 0.014002 & 0.017307 \\
\hline 2 & 0.014002 & 0.017307 \\
\hline 3 & 0.01181 & 0.016505 \\
\hline 4 & 0.01181 & 0.016505 \\
\hline 5 & 0.012324 & 0.016661 \\
\hline 6 & 0.012324 & 0.016661 \\
\hline 7 & 0.012136 & 0.016605 \\
\hline 8 & 0.012136 & 0.016605 \\
\hline 9 & 0.012324 & 0.016632 \\
\hline 10 & 0.012324 & 0.016632 \\
\hline 11 & 0.012176 & 0.016617 \\
\hline 12 & 0.012176 & 0.016617 \\
\hline
\end{tabular}

TABLE 4: Converged solutions for bending moments at the center of rectangular Kirchhoff CSCS plate under hydrostatic loads

$$
p(x)=p_{0} x / a
$$

\begin{tabular}{|c|c|c|c|c|}
\hline \multirow{2}{*}{$b / a$} & \multicolumn{2}{|c|}{ Present study } & \multicolumn{2}{c|}{ Timoshenko and Woinowsky-Krieger } \\
\cline { 2 - 5 } & $M_{x x}$ & $M_{y y}$ & $M_{x x}$ & $M_{y y}$ \\
\hline 0.50 & $0.007 p_{0} b^{2}$ & $0.021 p_{0} b^{2}$ & $0.007 p_{0} b^{2}$ & $0.021 p_{0} b^{2}$ \\
\hline 0.75 & $0.011 p_{0} b^{2}$ & $0.020 p_{0} b^{2}$ & $0.011 p_{0} b^{2}$ & $0.021 p_{0} b^{2}$ \\
\hline 1.0 & $0.012 p_{0} a^{2}$ & $0.017 p_{0} a^{2}$ & $0.013 p_{0} a^{2}$ & $0.017 p_{0} a^{2}$ \\
\hline 1.25 & $0.021 p_{0} a^{2}$ & $0.021 p_{0} a^{2}$ & $0.021 p_{0} a^{2}$ & $0.021 p_{0} a^{2}$ \\
\hline 1.50 & $0.030 p_{0} a^{2}$ & $0.023 p_{0} a^{2}$ & $0.030 p_{0} a^{2}$ & $0.023 p_{0} a^{2}$ \\
\hline 2 & $0.043 p_{0} a^{2}$ & $0.024 p_{0} a^{2}$ & $0.043 p_{0} a^{2}$ & $0.024 p_{0} a^{2}$ \\
\hline$\infty$ & $0.063 p_{0} a^{2}$ & $0.019 p_{0} a^{2}$ & $0.063 p_{0} a^{2}$ & $0.019 p_{0} a^{2}$ \\
\hline
\end{tabular}

TABLE 5: Converged solutions for Kirchhoff (CSCS) plate under hydrostatic load $p(x)=p_{0} x / a, n=11$

\begin{tabular}{|c|c|}
\hline$b / a$ & $w_{c}\left(p_{0} a^{4} / D\right)$ \\
\hline 0.5 & $8.1576 \times 10^{-5}$ \\
\hline 1.0 & $9.5855 \times 10^{-4}$ \\
\hline 2 & $4.2224 \times 10^{-3}$ \\
\hline
\end{tabular}

\section{DISCUSSION}

The Kantorovich-Vlasov method has been successfully implemented to solve the boundary value problem of Kirchhoff (CSCS) plates under linearly distributed transverse load $p(x)=p_{0} x / a$, over the entire plate domain. The boundary value problem was presented in variational form so that the problem became one of finding the deflection function that minimized the total potential energy functional. Based on Vlasov and Kantorovich approaches, the deflection function was considered as Equation (4). This resulted in the total potential energy functional given by Equation (9). The Euler-Lagrange-Ostragradsky differential equation for the problem was obtained as Equation (13) for any distribution of applied transverse load and Equation (18) for the specific case of linearly distributed transverse load considered in this study. The general solution to the Euler-Lagrange-Ostragradsky equation was obtained as Equation (31) using the linearity principles for solving non-homogeneous ordinary differential equations. Boundary conditions were enforced to obtain the general solution for the deflection function as Equation (56) where the integration constants were obtained in terms of the plate aspect ratios as Equations (57) - (60). Bending moment curvature relations were used to obtain the 
bending moment distributions $M_{x x}$ and $M_{y y}$ from Equations (61), (62), (63) and (64). The deflection and bending moment expressions were found to be single series expressions. Values of the deflection and bending moments were calculated for various aspect ratios of the plate and presented in Tables 2, 4 and 5. The center deflections and bending moments for square Kirchhoff CSCS plate under the linearly distributed load considered were also obtained for various terms of the single series and presented in Tables 1 and 3 in order to study the convergence properties of the series for deflection and the series for bending moment. Table 1 shows that the series for deflection converges rapidly and reasonably accurate results for deflection are obtained by using $n=5$. Similarly, the series for bending moments converge less rapidly, but reasonably accurate results are obtained for square plates using $n=7$ in the series for both $M_{x x}$ and $M_{y y}$. Tables 2, 3 and 4 which present converged results for deflection and bending moments for various plate aspect ratios illustrate the good agreement between the results of the present study and Timoshenko and Woinowsky-Krieger's solution for the same problem. Timoshenko and Woinowsky-Krieger obtained their solutions by linear superposition of the solution for simply supported rectangular Kirchhoff plate under the linearly distributed transverse load $p(x)=p_{0} x / a$ with the solution for the same problem under an applied distribution of torque along the fixed edges ( $y=0$ and $y=b$ ), where the torque is of such a magnitude that rotational displacement is fully restrained at the fixed edges.

\section{CONCLUSIONS}

From the study, the following conclusions are made:

(i) the Kantorovich-Vlasov method can be successfully implemented for the solution of the rectangular Kirchhoff CSCS plate under linearly distributed transverse load on the entire plate region

(ii) the solutions obtained for the deflection $w(x, y)$ and bending moment expressions $M_{x x}$ and $M_{y y}$ are single series containing exponential functions

(iii) the series for deflection and bending moment expressions are convergent but the series for deflection is more rapidly convergent than the series for bending moments

(iv) the converged results obtained for the deflections and bending moments at the center of the plate for various plate aspect ratios agree excellently well with the solutions presented by Timoshenko and Woinowsky-Krieger.

\section{REFERENCES}

[1] K. Chandrashekhara “Theory of Plates,” University Press (India) Limited, Hyderabad, 2011.

[2] R. Szilard "Theories and Applications of Plate Analysis: Classical, Numerical and Engineering Methods,” John Wiley and Sons Inc. 2004.

[3] S. Timoshenko and S. Woinowsky-Krieger “Theory of Plates and Shells,” 3rd Edition, McGraw Hill Book Co. New York, 1959.

[4] E. Reissner "The effect of transverse shear deformation on the bending of elastic plates,” Journal of Applied Mechanics, Vol 12, pp. 69-77, 1945.

[5] R.D. Mindlin "Influence of rotary inertia and shear on flexural motions of isotropic, elastic plates,” J. App. Mech. Vol 18, No 1, pp 3138, 1951.

[6] R.P. Shimpi “Refined plate theory and its variants,” AIAA Journal Vol 40, No 1, pp. 137-146, January 2002.

[7] Y. Suetake "Plate bending analysis by using modified plate theory,” CMES Vol 11, No 3, pp 103-110, Techno Science Press. 2006.

[8] C.L.M.N. Navier "Bulletin des science de la societie philomartrique de Paris,” 5 pp 95-102, Extrait des recherché sur la flexion des planes elastiques, 1823.

[9] M. Levy "Memoire sur la theorie des plaques elastiques planes,” Journal de Mathematiques Pures et Appliqueés, Vol 30, pp. 219-306, 1899.

[10] J.C. Ezeh, O.M. Ibearugbulem and C.I. Onyechere "Pure bending analysis of thin rectangular flat plates using ordinary finite difference method,” International Journal of Emerging Technology and Advanced Engineering (IJETAE) Volume 3, Issue 3, pp. 2023, March 2013.

[11] C.H. Aginam, C.A. Chidolue and C.A. Ezeagu "Application of Direct Variational Method in the Analysis of Isotropic, thin, rectangular plates,” ARPN Journal of Engineering and Applied Sciences, Vol 7 No 9. Asian Research Publishing Network pp. 11281138. September 2012.

[12] N.N. Osadebe, C.C. Ike, H.N. Onah, C.U. Nwoji and F.O. Okafor "Application of the Galerkin-Vlasov method for the analysis of simply supported rectangular Kirchhoff plates under uniform loads,” Nigerian Journal of Technology (NIJOTECH), Vol 35, No 4, pp 732-738, October 2016.

[13] C.U. Nwoji, B.O. Mama, C.C. Ike and H.N. Onah "Galerkin-Vlasov method for the flexural analysis of rectangular Kirchhoff plates with clamped and simply supported edges,” IOSR Journal of Mechanical and Civil Engineering (IOSR JMCE) Volume 14, Issue 2, Version 7, pp 61-74, March 2017.

[14] C.U. Nwoji, B.O. Mama, H.N. Onah and C.C. Ike "Kantorovich-Vlasov method for simply supported plates under uniformly distributed loads,” International Journal of Civil, Mechanical and Energy Science (IJCMES) Volume 3, Issue 2, pp 69-77, March-April 2017.

[15] C.C. Ike “Kantorovich-Euler Lagrange-Galerkin method for bending analysis of thin plates,” Nigerian Journal of Technology, NIJOTECH, Volume 36, No 2, pp 351-360, April 2017. 


\section{Author Profile}

Hyginus Nwankwo Onah (B.Eng, M.Eng, PhD) Born in Nigeria on May $4^{\text {th }} 1959$, I obtained my B.Eng. (Civil Engineering) in 1983. After brief work in Lagos Nigeria, I travelled to France in 1987 where I obtained M.Eng (Civil Engineering) at INSA de Lyon and Ph.D (Structural Mechanics) at CNAM Paris in 1991. Then I worked as a researcher at Research and Development (R\&D) unit of RENAULT Motors Reuil Malmaison France, Department of Engineering University of Manchester England and Becchis Osiride of FIAT Motors Torino Italy for one year each. From January 1996 to date as a lecturer in Civil Engineering Department of University of Nigeria, Nsukka, I teach and supervise both undergraduate and Postgraduate students and carry out research activities in mechanics and behavior of structures

Engr Dr Benjamin Okwudili Mama I got my PhD in the year 2009 in the University of Nigeria, Nsukka. My master's degree in civil Structural Engineering in 1992 in the same School. My first degree in Civil Engineering in then Anambra State University of Technology, Enugu. I have been lecturing in the Department of Civil Engineering, University of Nigeria, Nsukka from 1994 till date.

Engr Dr. Charles C. Ike is a lecturer at the Enugu State University of Science and Technology, Enugu, Nigeria. He holds a $\mathrm{PhD}$ in structural engineering, and is a member of the Nigerian Society of Engineers. He is registered with the Council for the Regulation of Engineering in Nigeria (COREN). He has numerous publications in reputable international journals.

Engr Dr. Clifford U. Nwoji obtained his civil engineering education from University of Nigeria Nsukka. He is a member of Nigerian Society of Engineers and also a registered engineer with Council for Regulation of Engineering in Nigeria. He has substantial industrial experience. Since obtaining his higher degrees in engineering he has been teaching structural engineering courses at both undergraduate and postgraduate levels. He has successfully supervised higher degree theses. He has to his credit a good number of published research papers in reputable international journals. 